\title{
A model for understanding diagnostic imaging referrals and complex interaction processes within the bigger picture of a healthcare system
}

\author{
Chandra R. Makanjee ${ }^{\mathrm{a}, *}$, Anne-Marie Bergh ${ }^{\mathrm{b}}$, Willem A. Hoffmann ${ }^{\mathrm{c}}$
}

\author{
${ }^{a}$ Department of Radiography, University of Pretoria, Private Bag X323, Arcadia 0007, South Africa \\ ${ }^{\mathrm{b}}$ MRC Unit for Maternal and Infant Health Care Strategies, University of Pretoria, South Africa \\ ${ }^{\mathrm{c}}$ Department of Biomedical Sciences, Tshwane University of Technology, Private Bag X680, Pretoria 0001, South Africa
}

\begin{abstract}
A b s t r a c t
Using experiences from the South African public healthcare system with limited resources, this review proposes a model that captures a holistic perspective of diagnostic imaging services embedded in a network of negotiated decision-making processes. Professional interdependency and interprofessional collaboration, cooperation and coordination are built around the central notion of integration in order to achieve a seamless transition through the continuum of various types of services needed to come to a diagnosis. Health-system role players interact with patients who enter the system from the perspective of their life-world. The distribution of diagnostic imaging services - within one setting or at multiple levels of care - demonstrates how fragments of information are filtered, interpreted and transformed at each point of care. The proposed model could contribute to alignment towards a common goal: services providing holistic quality of care within and beyond a complex healthcare system.
\end{abstract}

Keywords:

Health system

Delivery of healthcare

Patient care team

Referral and consultation

Integration

Interpretation

\section{Introduction}

Referrals for a diagnostic imaging investigation require the request for an opinion from a radiology specialist to detect or exclude disease. This action contributes to the prognosis, patient management, monitoring of the clinical course of disease and/or screening for at-risk health. ${ }^{1,2}$ The referral and the imaging investigation itself lie "in the middle of the continuum" ${ }^{3}$ of the patient's journey through the healthcare system.

The aim of this review article is to provide a holistic perspective of diagnostic imaging services in a resource-limited healthcare system, based on the authors' healthcare research experience in South Africa. The proposed model incorporates medical- and nonmedical-provider interaction processes mediated through text (documents and files) and technology. The model could form the basis for research aimed at providing a holistic account of the quality of care services in a healthcare setting. The focus also includes a way of understanding how the lower levels of care in the healthcare sector, with a limited choice of imaging modalities, interact with higher levels of care.

\footnotetext{
* Corresponding author. Tel.: +27 123541236.

E-mail address: Chandra.Makanjee@up.ac.za (C.R. Makanjee).
}

Diagnostic imaging within the complex healthcare system

The division between public and private health sectors is a global arrangement. Many countries, just as in South Africa, divide their public sector into the following levels: primary (level 1, district hospital), secondary (level 2, regional hospital) and tertiary (level 3, central hospital), ${ }^{4}$ with specific guidelines for referrals between these levels. District hospitals often provide only X-ray services, and in some instances basic sonography and fluoroscopy. Regional hospitals provide additional services such as computed tomography (CT) and diagnostic sonography services, whereas central or tertiary-level hospitals provide access to the whole spectrum of diagnostic imaging services. Fig. 1 provides an overview of referral pathways and flows of processes for diagnostic imaging investigations in the public sector.

There is a wide diversity among countries regarding the structuring of the private health sector. In South Africa it is, for example, divided into a large, corporate private-for-profit hospital sector and a smaller and diversified, private-not-for-profit sector. ${ }^{4,5}$ No complete typology of private and industry or workplace hospitals is available. Radiological services are outsourced, regardless of the level of the hospital. In the private sector patients often have direct access to some specialised imaging services via their general practitioners. 


\section{LEVELS OF CARE}

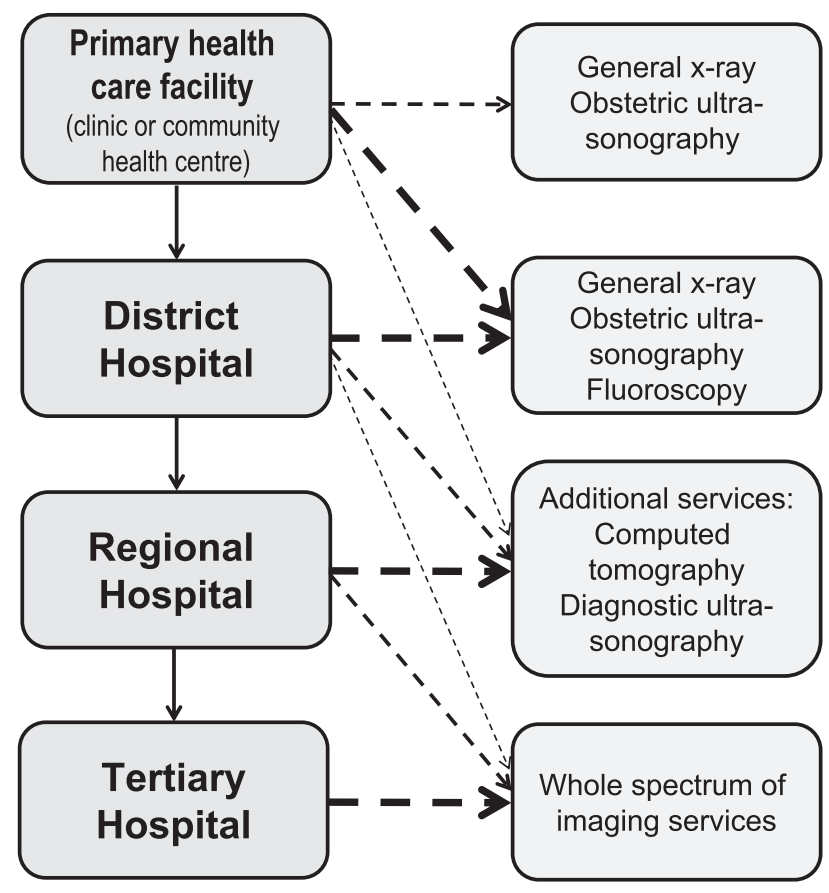

$\downarrow \begin{aligned} & \text { Normal clinical referral } \\ & \text { pathway of patients }\end{aligned}$

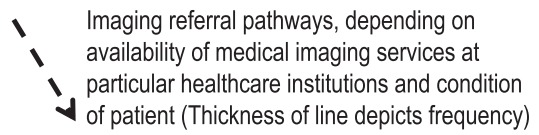

Figure 1. Referral pathways for diagnostic imaging services in the public sector.

The focus of this paper is mainly on diagnostic imaging in the public sector of a resource-limited country. However, client cross-over between the public and private sectors when seeking healthcare is a common phenomenon. This type of fragmentation impacts on the healthcare system and on patients - financially and clinically - in terms of providing a continuum of services of optimal quality. Although diagnostic imaging (including referral, decision making and interaction) focuses on individual patients at a micro-level in the healthcare system, meso- and macro-level trends influence the individual encounters between patients and healthcare providers and the outcomes with regard to management, treatment, and follow up. ${ }^{6,7}$

When reflecting on diagnostic imaging services as being embedded in and intertwined with the medico-clinical pathways, the notion of integration becomes central. Integration implies effective, accessible, continuous and comprehensive care services of quality. It also entails collaboration, coordination and alignment of interactions and decisions, mediated through healthcare providers. These providers are, according to the actor network theory, first-order actors, with text and technology being second-order actors. ${ }^{8}$ Collective intra- and inter-institutional navigation is required to provide the necessary continuum of care and a smooth and safe transition for the patient in his or her journey through the healthcare system until the ultimate diagnosis and treatment plan can be achieved. ${ }^{8-11}$

\section{Complexity and interconnectivity in interactions and decision- making processes}

Each service stage of the patient's journey is made up of multiple small steps with interfaces between these steps. Caring for an individual patient requires a holistic un-derstanding, greater than the sum of its parts. The 'bigger picture' emerges from the complexities and interactions in a health system. The building blocks for making a decision regarding the most appropriate diagnostic investigation of choice with minimal risk and optimal benefit are strongly shaped by organisational structure, institutional members, the quality of information on the request document, the actual investigation and its outcomes. The process evolves as the various events unfold, based on the actions taken with each event and depending on the interactions of the professionals within the system. The process is highly dependent on who is communicating with whom in a particular institution or what forms the content of the communication.

The hierarchical interconnectivity between different levels of the health system ${ }^{12}$ is evident throughout the referral process for diagnostic imaging investigations. A hospital is an indivisible unit with a high degree of specialisation and diversity among its healthcare providers, its components (including technology), and feedback and interaction loops. ${ }^{13}$

Decisions in everyday situations are embedded in a broader context and are part of a decision-action cycle that is affected by monitoring and feedback and not by a single judgement. ${ }^{10}$ The way that services are distributed in the healthcare context of diagnostic imaging often means that the notion of interprofessional, teambased, patient-centred decision making does not apply or is not appropriate. ${ }^{14}$ In this regard, Patel et al. ${ }^{13}$ refer to distributed diagnostic imaging services involving the "management of streams of information and ... communication and coordination among individuals and from data sources" in order to come to shared understandings and decisions. Meaningful communication relationships between healthcare professionals and between professionals and patients are difficult to develop without mutual understanding. ${ }^{10}$ The interdependency at different levels and stages of decision making and the diversity of healthcare providers involved in the fragments of decisions contribute towards building the bigger picture. "Even if a single individual ultimately decides on a course of action others are critically involved in the process". 13

We developed a model to capture the bigger, institutional macro-level picture applicable at any hospital or health centre providing diagnostic imaging services. The model depicted in Fig. 2 was adapted from Yabroff's ${ }^{15}$ health services research framework for evaluating cancer screening. It shows the interconnectivity between healthcare professionals (including various levels of the healthcare system) and patients. It also shows the network of relations and interactions between the role players involved in referrals for diagnostic imaging investigations, interpretation of images, communication of results, and the drafting of treatment and management plans.

At the macro-level the model contextualises the connectivity of the health system and its providers with patients and their communities regarding decision making in diagnostic imaging. The common goal shared by all healthcare providers is to deliver quality service and care to the patient. ${ }^{16}$ Central to this network of interconnectivities are what patients present with and how they present their histories, their symptoms and the narratives of their condition. The immediate challenge is the coordination of information as part of a continuum of patient care within this network of interactions.

\section{Routine actions and interactions in decision making}

Various role players routinely interact in decision making at the meso- and macro-level. Diagnostic imaging routines are highly subject to context, needing adaptation as the context or situation changes. ${ }^{17}$ However, the quality of routine actions should continue to enable continued efficiency and optimal outcomes in all circumstances. 


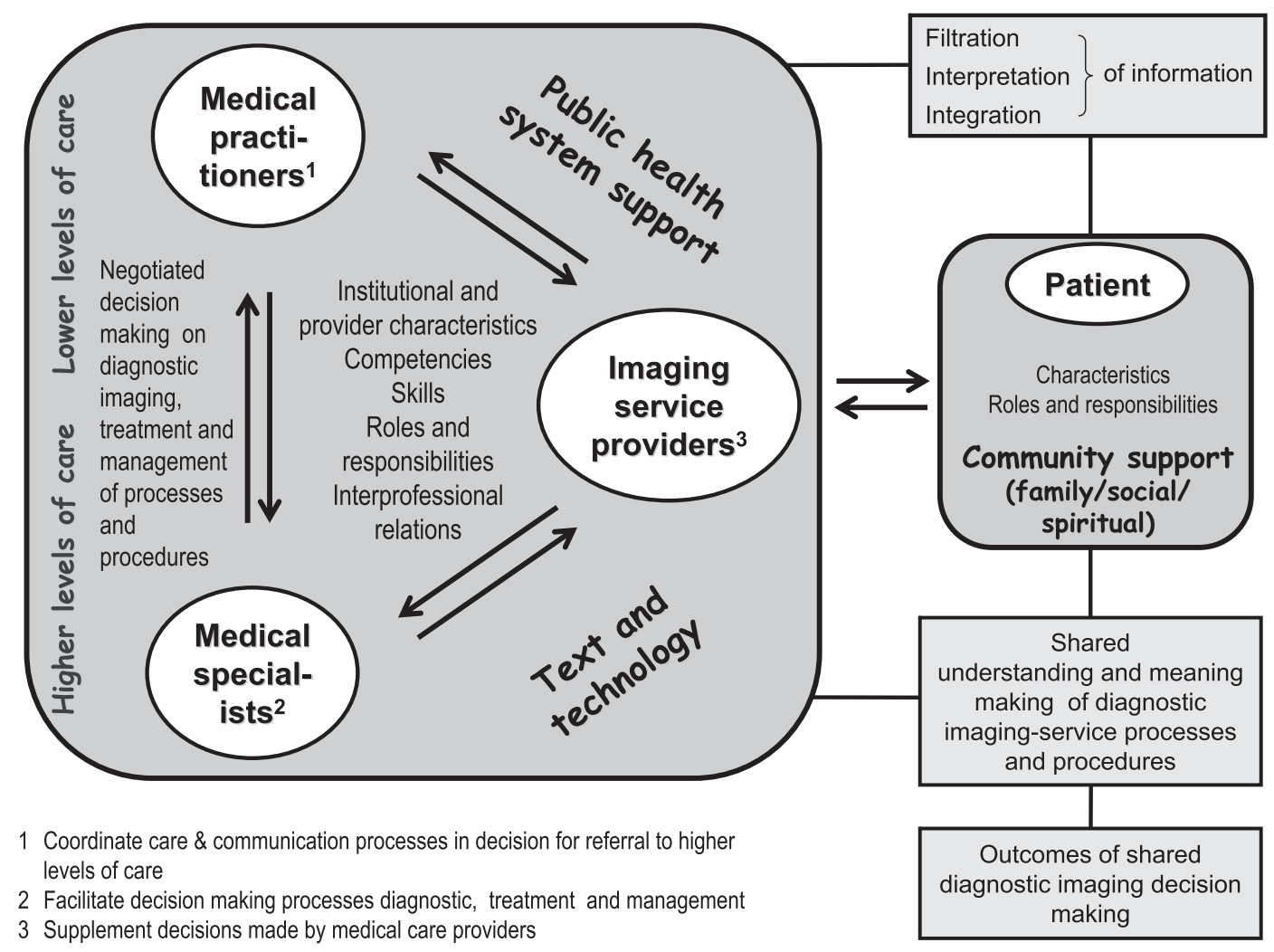

Figure 2. Role players and inter-level interactions in decision making around diagnostic imaging.

In the South African public health sector routine actions to access some specialised imaging modalities entail building up clinical evidence to justify a request. The complexity of a case determines the progress through the levels of care, from the district to the regional to the tertiary hospital (Fig. 1 ). ${ }^{18}$ This depends, inter alia, on the severity of the condition, the accessibility of services and technologies, and the availability of expertise required for management and treatment. ${ }^{19}$ This is in contrast with the private sector, where financial incentives for the provider via payments by medical insurance and the consumerist approach result in easier access to a specialist and a range of modalities. ${ }^{18}$

A typical example of a complex case is the referral pathway for patients with cervical spine trauma admitted at the casualty department of a district hospital in South Africa. Contrary to the prescribed referral pathway, such a patient does not always have to be examined by a specialist before being referred for a CT scan at the second or third level of care. Based on the interpretation of plain images, the referring medical practitioner can request an orthopaedic surgeon to authorise the referral for a scan before the practitioner contacts the radiologist directly.

Within the confines of any hospital level, medical care providers (general practitioners and family physicians) and radiographers providing plain radiographic imaging services interact with each other, often in a transactional manner. Fig. 2 illustrates the healthcare-provider interactions between different levels of care in the public health system. It provides a better understanding of the rigid "ceremonial" 20 processes within which healthcare providers find themselves. In other words, it is the unwritten, prescribed way of behaviour that shapes decision-making and interaction pathways that public-sector providers adhere to.

However, in our experience medical practitioners at some district hospitals view the bureaucratic system of the higher levels of healthcare as an obstacle for referrals to the appropriate modalities of diagnostic imaging investigations, as well as to access expert radiological opinion. There is also a perception that medical officers (general practitioners) working in regional and tertiary hospitals have easier access to these sophisticated modalities and expert opinions than practitioners in district hospitals. The reality is, however, that access to certain modalities at higher levels of care is restricted by a step-by-step process, starting with referrals for basic examinations, followed by a referral from a general to a specialised department, even within the same level of care. This process does not necessarily correspond to the ideal of making an accurate diagnosis by means of direct access to the most appropriate modality for providing clinical evidence.

A typical example of accessibility is the referral route for a mammography investigation in the South African public sector. The district hospitals' medical officers are unable to refer directly, in contrast to their counterparts in the private health sector. Medical officers in the public sector have to follow a rigid step-by-step process, starting with a physical examination followed by a fine needle aspiration, then a biopsy and ultimately a mammogram. In view of the absence of a general mammography screening programme in South Africa, together with limited access to mammography, CT and magnetic resonance imaging (MRI) services at most public hospitals, serious questions could be raised about timely diagnosis, patient safety, cost efficiency, risks to patients and the impact of the anxiety created in patients.

Patients who enter the health system bring with them a lifeworld (including a community system of support) (Fig. 2). This impacts on their micro-level interactions with different healthcare providers at each point of contact in their journey through the system. These interactions are often very brief encounters, with each encounter resulting in the classification of patients into specific hierarchies. As a result, diagnoses of increasing severity determine the journey of some patients to higher levels of the system. ${ }^{18}$ 
All role players (groups and individuals) bring with them specific personal characteristics, skills, competencies, roles and responsibilities (Fig. 2). The main responsibility of medical-care providers at all levels is to coordinate and manage patient care, as well as treatment processes and procedures. Diagnostic imaging service providers (radiographers and radiologists) and nurses play a supplementary role. Their main function is to support the medical practitioner in diagnostic decision making by rendering a professional service of high quality, specifically by means of diagnostic imaging investigations and radiological reports.

The medical practitioner is the coordinator of the referral processes in hospital settings at the lower levels of care (Fig. 2). ${ }^{21}$ One of the most important skills or characteristics related to a referral for a diagnostic image is knowledge about the most appropriate investigation to assist in making a diagnostic decision. An effective decision requires the practitioner to be able to evaluate the quality of an image, to interpret the image, and to integrate the information of the radiological report into the bigger clinical picture.

\section{Filtering, interpretation and integration of information}

The ability to use and transform information in order to come to a diagnosis and formulate a treatment plan is an important competency required of medical care providers. Their task is supplemented by high-quality radiographic images and radiological reports provided by radiographers and radiologists, respectively (Fig. 2). On the one hand, the quality of a diagnostic imaging investigation is dependent on the radiographer's ability to make a judgement on the appropriateness of the investigation. Making such a judgement is in turn dependent on the quality of information on the request form (provided by the medical care provider), the cooperation of the patient and the available imaging technology. On the other hand, the radiologist relies on the quality of information on the request form and on the quality of the images (provided by the radiographer) to enable integration and to make an interpretational judgement regarding the diagnosis or differential diagnosis. ${ }^{22}$

Within the bigger picture of decision-making and diagnostic processes, information and knowledge input and output at different points characterise the patient's journey through the healthcare system. These actions form the basis of decision-making processes $^{23,24}$ that could lead to improved operational, economic or clinical benefit. ${ }^{25}$ Interpretation also draws on mediation tools such as the request form and other technological informationprocessing systems, which are embedded in contexts such as work practices, organisational structures and cultures, and interpersonal relations. ${ }^{26}$

In order to construct and reconstruct a diagnosis and a management plan to improve patient outcomes, information in the form of empirical evidence - the 'right' piece of information needs to be gathered during a medical consultation and by means of diagnostic tests. ${ }^{27,28}$ Everitt $^{27}$ describes the pivotal questions as: "what information to gather; which diagnostic test to perform; how to interpret and integrate this information to draw diagnostic conclusions". As such, the quality of information is highly dependent on the nature and quality of provider-patient and providerprovider interactions mediated through documentation (text) and technology. The practice of information exchange is an important feature where information gathering, processing and verification are concerned - especially where technological information has to be transformed into biomedical concepts and narratives that form part of the professional practices of medical practitioners. In this regard, Eisenberg and colleagues ${ }^{29}$ refer to the critical role of professionals in filtering cues. They have to process the cues and decide which to ignore or bracket, ${ }^{26}$ which to translate into a list of actions, or which to integrate into the final diagnosis. Data and information are therefore filtered in a sequence of transactions through diverse sources (providers and patients) in an effort to make sense of how to interpret, ${ }^{17}$ integrate and transform ${ }^{3}$ information in a meaningfully connected and coherent manner. ${ }^{17}$

\section{Alignment of patient and healthcare provider goals}

The alignment of goals that takes into consideration patient expectations and preferences is part of the diagnostic imaging process. ${ }^{16,30}$ This entails obtaining adequate information for decision-making activities and actions by means of interactive communication in order to enhance both medical and lay understanding. ${ }^{31}$ The image itself provides visibility and concreteness, enabling the alignment between patients and healthcare providers. It also demonstrates alignment with reality. ${ }^{32}$

Patients have specific roles and responsibilities within the public healthcare system (Fig. 2): to provide accurate information about their illness and/or concerns or previous medical encounters; to take interest in their health and well-being; to inform healthcare providers of their expectations in terms of outcomes; and, in some countries, to keep imaging and/or previous discharge records. ${ }^{33}$ In some settings patients generally play a relatively passive role in the provider-patient interaction. They also rely on healthcare professionals to effectively communicate with them, particularly before a diagnosis, when there is uncertainty and fear regarding the experienced symptoms. Patient experiences of illness can extend to a wider context beyond the boundaries and settings of formal diagnosis and treatment, ${ }^{34}$ and impact on their experience of, for example, quality of life. ${ }^{30}$

\section{Negotiated decision making}

Diagnostic imaging investigations are embedded in a network of negotiated decision-making processes. This is especially evident where one type of healthcare provider or profession needs the services or expertise of another or where technology (e.g. diagnostic imaging equipment or computers) contributes to the transformation of information in a significant way. ${ }^{23}$ These kinds of decisions are rarely based on team work, as they are outcomes of temporary interactions with other healthcare providers. Bleakley ${ }^{14}$ refers to "teeming", "negotiated knotworking" (networking) and "collaborative intentionality" to describe the transience of these interactions. Where diagnostic imaging investigations are at stake, negotiated decision making often takes places at discreet moments in the unfolding of decision-making events.

Healthcare providers also have to negotiate with patients at various points of contact before coming to a final diagnosis and treatment decision. Where more than one treatment or investigation option is available or where a patient needs more time to give permission for an investigation or treatment, behavioural outcomes - following certain decisions that have been made - are constructed from negotiations. ${ }^{23}$ In addition, patient-centred care recognises the asymmetries in the status of healthcare providers and patients, which should be resolved by involving all role players to cooperate, collaborate and coordinate through negotiations rather than by the prescribed rules of behaviour. ${ }^{22,35,36}$

\section{Conclusion}

To the best of our knowledge, this is one of the first attempts to describe the referral system and interaction processes in diagnostic imaging in the public-sector context of a developing country with limited resources. The South African healthcare system was used as the entry point to highlight challenges in providing timely access to 
specialised diagnostic imaging services and specialist care, as illustrated by the inadequacies of referral for mammography, MRI and CT scans. These have consequences for quality healthcare to patients. The dilemmas point towards the need to review the distribution of diagnostic imaging services, the way in which catchment areas for patient admissions and referrals is prescribed, and the types of patients that access services at particular points of healthcare.

The perspectives presented could also be applicable to other countries with similar constraints and limited access to specialised medical imaging services. Despite suboptimal and sometimes risky referral mechanisms available to access technical healthcare services, the review of the healthcare system enabled us to make visible the referral pathways and interaction processes aimed at accessing diagnostic imaging services. The model provides a point of departure for interrogating the adequacy of current practice, routine actions and interaction processes between the role players in a multidisciplinary team. It also facilitates the review of coordination, collaboration and cooperation in a hierarchical health system, from referral to outcomes. Further research studies should be conducted in other countries with similar healthcare systems to validate, amend or modify the various elements of the proposed model.

\section{Conflict of interest statement}

The authors declare they have no conflicts of interest.

\section{Funding}

There were no sources of funding for this study.

\section{Author contributions}

All three authors contributed to the conceptualisation and refinement of the model. The first author wrote the first draft of the article, on which the other two authors gave critical input. All authors approved the final manuscript for submission.

\section{Acknowledgements}

The authors wish to thank the following individuals: all the colleagues and patients who contributed to the conceptualisation of the model and Barbara English (Office of the Deputy Dean: Research, Faculty of Health Sciences, University of Pretoria) who provided editorial support; and the reviewers for their valued comments that further enhanced the quality of the manuscript.

\section{References}

1. European Commission, Directorate-General for the Environment Radiation protection 118. Referral guidelines for imaging. Luxembourg: Office for Official Publications of the European Communities; 2001. http://www.ec.europa.eu/ energy/nuclear/radioprotection/publication/doc/118_en.pdf [accessed 2007].

2. Knottnerus JA, van Wheel C, Muris JWM. Evidence base of clinical diagnosis: evaluation of diagnostic procedures. BMJ 2002;324:477-80.

3. Boon HS, Mior SA, Bamsley J, Ashbury FD, Haing R. The difference between integration and collaboration in patient care: results from key informant interviews working in multiprofessional healthcare teams. J Manip Physiol Ther 2009;32(9):715-22.

4. Republic of South Africa. Government Notice R. 655: National Health Act (61/ 2003): regulations: categories of hospitals. Gov Gaz 2011;554(34521):3-27 (Regulation Gazette No. 9570, 12 August 2011).

5. Heunis JC. Hospitals and hospital reform in South Africa. In: Van Rensburg HCJ, Benator SR, Doherty JE, Heunis JC, McIntyre DE, Ngwena CG, et al., editors. Health and healthcare in South Africa. 1st ed. Pretoria: Van Schaik; 2004. pp. 459-505.
6. Charles C, Gafni A, Whelan T, O' Brien MA. Cultural influences on the physicianpatient encounter: the case of shared treatment decision-making. Patient Educ Couns 2006;63(3):262-7.

7. Van Rensburg HCJ, Pelser AJ. The transformation of the South African health system. In: Van Rensburg HCJ, Benator SR, Doherty JE, Heunis JC, McIntyre DE, Ngwena CG, et al., editors. Health and health care in South Africa. 1st ed. Pretoria: Van Schaik; 2004. pp. 149-50.

8. D’Amour D, Goulet L, Labadie JF, San Martín-Rodriquez L, Pineault R. A model and typology of collaboration between professionals in healthcare organizations. BMC Health Serv Res 2008;8(188):1-14.

9. Longo J, Smith MC. A prescription for disruptions in care: community building among nurses to address horizontal violence. Adv Nurs Sci 2011;34(4):345-56.

10. Suter E, Arndt J, Arthur N, Parboosingh J, Taylor E, Deutschlander S. Role understanding and effective communication as core competencies for collaborative practice. J Interprof Care 2009;23(1):41-5.

11. Clark FA. Power and confidence in professions: lessons for occupational therapy. Can J Occup Ther 2010;77(5):264-9.

12. Weiner SJ. Contextualizing medical decisions to individualize care lessons from the qualitative sciences. J Gen Intern Med 2004;19(3):281-5.

13. Patel VL, Kaufman DR, Arocha JF. Methodological review: emerging paradigms of cognition in medical decision-making. J Biomed Inform 2002;35(1):52-75.

14. Bleakley A. Professing medical identities in the liquid world of teams. Med Educ 2006;45(12):1167-73.

15. Yabroff KR. Interventions to improve cancer screening: commentary from a health service research perspective. Am J Prev Med 2008;35(Suppl. 1):S6-9.

16. Feldman-Stewart D, Brundage MD, Tishelman C. A conceptual framework for patient-professional communication: an application to the cancer context. Psycho Oncol 2005;14(10):801-9.

17. Hilligoss PB. Patient handoffs between emergency department and inpatient physicians: a qualitative study to inform standardization of practice and organization theory. Doctoral thesis in Information submitted to the University of Michigan, http://deepblue.lib.umich.edu/bitstream/2027.42/86293/1/bhilligo_ 1.pdf; Feb 2011 [accessed 2012].

18. Gibson D Negoatiating the new health care system in Cape Town: five case studies of the actually chronically ill. Med Anthropol Q 2001: 15(4):515-32.

19. Geneau R, Lehoux $P$, Pineault $R$ Understanding the work of general practitioners: a social science perspective on the context of medical de-cision making in primary care. BMC Fam Pract 2008;9(12):1-10.

20. Staniland K. Studying organizations: the revival of institutionalism. In: Bourgeault I, Dingwall R, De Vries R, editors. The SAGE handbook of qualitative methods in health research. 1st ed. London: Sage; 2010. pp. 249-65.

21. Coyle E, Hanley K, Sheerin J. Who goes where? A prospective study of referral patterns within a newly established primary care team. Ir J Med Sci 2011;180(4):845-9.

22. Yedidia MJ. Transforming doctor-patient relationships to promote patientcentered care: lessons from palliative care. J Pain Symptom Manage 2007;33(1):40-56.

23. Wilson SJ. The myth of objectivity: is medicine moving towards a social constructivist paradigm? Fam Pract 2000;17(2):203-9.

24. Whiting P, Toeriena M, De Salisa I, Sternea JAC, Dieppea P, Eggerb M, et al. A review identifies and classifies reasons for ordering diagnostic tests. J Clin Epidemiol 2007;60(10):981-9.

25. Price P. Evidence-based laboratory medicine: supporting decision-making. Clin Chem 2000;46(8):1041-50.

26. Paul SA. Understanding together: sensemaking in collaborative information seeking. Doctoral dissertation in Information Sciences and Technology submitted to the Pennsylvania State University, http://www.personal.psu.edu/ sap246/spaul_CISWorkshop_Final.pdf; May 2010 [accessed 2103].

27. Everitt S. Clinical decision making in veterinary practice. Doctoral thesis submitted to the University of Nottingham, http:// etheses.nottingham.ac.uk/2051/; 2011 [accessed 2012].

28. Tatsioni A, Zarin DA, Aronson N, Samson DJ, Flamm CR, Schmid C, et al. Challenges in systematic reviews of diagnostic technologies. Ann Intern Med 2005;142(12 Part 2):1048-55

29. Eisenberg EM, Murphy AG, Sutcliffe K, Wears R, Schenkel S, Perry S, et al. Communication in emergency medicine: implications for patient safety. Comm Monogr 2005;72(4):390-413.

30. Halkett GKB, McKay J, Shaw T. Improving students' confidence levels in communicating with patients and introducing students to the importance of history taking. Radiography 2011;17(1):55-60.

31. Balaqué $F$, Cedraschi C. Radiological examination in low back pain patients: anxiety of the patient? Anxiety of the therapist? Jt Bone Spine 2006;73(5):508-13.

32. Rhodes LA, McPhillips-Tangum CA, Makham C, Klenk R. The power of the visible: the meaning of diagnostic tests in chronic pain. Soc Sci Med 1999;48(9): 1189-203.

33. Alfuth R, Barnard CP. Family physicians and family therapists: understanding the interdependent synergism. Contemp Fam Ther 2000;22(3):253-77.

34. Adams J, Smith T. Qualitative methods in radiography research: a proposed framework. Radiography 2003;9(3):193-9.

35. Kroenke K. Patient expectations for care: how hidden is the agenda? Mayo Clin Proc 1998;73(2):191-3.

36. Thiel De Bocanegra H, Gany F. Good provider, good patient: changing behaviors to eliminate disparities in healthcare. Am J Manag Care 2004;10:S20-8. 